\title{
Joint Cell Association and User Scheduling in Carrier Aggregated Heterogeneous Networks
}

\author{
Jia Zhang *, Lili Meng and Hui Ji
}

School of Information Science and Engineering, Shandong Normal University, Jinan 250358, China; menglili@sdnu.edu.cn (L.M.); hui.ji@sdnu.edu.cn (H.J.)

* Correspondence: zhangjia@sdnu.edu.cn; Tel.: +86-531-8618-0244

Received: 6 December 2017; Accepted: 31 December 2017; Published: 5 January 2018

\begin{abstract}
This paper focuses on the network capacity maximization problem through joint cell association and user scheduling with multiple carrier aggregation (CA) in the heterogeneous networks (HetNets). For the downlink transmission, the proposed joint maximization problem is reformulated from single data flow into multiple data flow through carrier aggregated HetNets, in which the users could associate with BSs on more than one carrier band. Such a flexible joint maximization problem could be solved by convex optimization solutions with reasonable complexity. Numerical analysis has confirmed the performance advantages of the proposed multi-flow solution under different carrier aggregation deployment.
\end{abstract}

Keywords: cell association; user scheduling; optimal resource allocation; HetNets; carrier aggregation

\section{Introduction}

Typical strategies are loosing the abilities to fulfill the growing demands of enormous wireless data rate and the heterogeneous density in wireless cellular networks for the 5th generation (5G) and beyond [1]. Heterogeneous networks (HetNets) have been considered as one of the most promising technologies for engaging better mobile coverage and higher cellular network capacity [2], which are developed by the Third Generation Partnership Project Long Term Evolution-Advanced (3GPP LTE-A) [3]. Transmitting nodes characterized by their graded coverage abilities within the HetNets are categorized into multiple layers accordingly to reinforce the service quality of mobile communications in a heterogeneous way [4].

As a new cellular paradigm, the deployment of small cells underlaid by a traditional macro cell is proved to be more efficient for communication due to the reduced transmission distance. However, the deployment of HetNets is challenging in several ways. For one thing, the coverage disparities of the transmit nodes in HetNets [5] would cause uneven traffic loads across different layers, which makes the users within the network more sensitive to the cell association policies. For another thing, massive small cell deployment would complicate the interference environment either among or across different layers, which would jeopardize the improvement of network spectral efficiency.

In order to cope with the unbalanced traffic load as well as the cross layer interferences, the problem of joint user scheduling and cell association must be further considered for the HetNets. To this end, this paper focuses on the network utility optimization of a two layer HetNet with multiple carrier bands fully reused across different network layers from a cell association and user scheduling angle.

\subsection{Related Work and Motivation}

Both industry and academia are convinced that the HetNets would play an important role in improving the cellular network capacity by extremely dense small cell BS deployments. However, such ultra density has made the network design more complicated in several ways. 
Intercell interference has always been a major concern for modern cellular networks. The LTE-A specifies several strategies for enhanced intercell interference coordination (eICIC), one of which is to explore cooperative [6] and coordinated transmission approaches involved with multiple antenna techniques [7-9]. Along with the ultra dense small cells deployment, massive antenna regime are considered the most crucial technologies in the $5 \mathrm{G}$ mobile systems $[10,11]$.

In the HetNets, challenges on interferences both intra-layer and inter-layer should be handled in a more spectrally efficient way that a successful cell deployment must offer a perfect balance between preferred network utility and the scarcity of radio resources. In the literature, a bunch of work has been done for spectral efficiency improvement in the HetNets by interference mitigation approaches [12,13]. Joint solutions of network MIMO are proved to have great potentials in interference management [14] for higher spectrum utilization.

To deal with the severe co-channel interference in the HetNets, another straightforward approach for the network design is the joint cell association and user scheduling problems aiming to maximize the overall network utility [15-17]. Traditional cellular scheduler associated the users with maximal signal to interference plus noise ratio (SINR) provider which may not be optimal, especially when multiple layer of small cells were engaged in the communication network [18-20]. One direct mend on this dilemma is to take the unbalanced traffic loads between the high transmit power layers and low power layers' BSs into account [21-23] when formulate the cell association problem based on max-SINR criterion. The small cell BS would benefit from such joint association and scheduling strategies from global optimization to locate the optimal cell load [24]. Moreover, game theory approaches [25,26] were also proved to offer the best negotiation on the cell range expansion balance among BSs with different transmit power level.

Most works in the literature are based on the assumption that all users associated on the same BS would share the power and other radio resources equally [27]. Full frequency reuse policy suffered heavily due to the dense deployment of small cells and the complicated interferences in the HetNets, where the spectral efficiency was already in jeopardy. In order to support high data rate and overcome the challenges on the spectrum scarcity in the same time, carrier aggregation (CA) has been introduced into future networks design [28,29]. Different frequency bands are involved in the downlink transmission of the HetNet with different coverage abilities and different propagation characteristics [30]. Under such circumstances, the joint problem of cell association and user scheduling in the HetNets would be more flexible if the users could be engaged with more than one carrier band with proper carrier aggregation strategies [31-33].

\subsection{Contribution and Organization}

The problem of joint resource allocation and user scheduling with carrier aggregation in current cellular networks were investigated in the literature [34,35]. However, very few were discussed when facing the dense deployments of small cells in the HetNets. In this context, the focus is on the network capacity optimization problem through joint cell association and user scheduling in a HetNet with multiple carrier aggregation across different layers. In such complicated communication environment, the original joint maximization on the network utility is formulated to be NP-hard. The main contribution of this work is providing an upper bound for the proposed joint problem through a nontrivial reformulation based on multi-flow carrier aggregation across the HetNet, which enables the users could associate with BSs over multiple carrier bands. Hence the joint maximization problem could be solved by convex optimization solutions with reasonable complexity. Under different carrier aggregation deployment, the performance advantages have been proved by numerical analysis.

The rest of this paper is organized as follows: Section 2 describes the system overview of the HetNet with both network and signal models, and formulates the problem of joint network capacity maximization. Section 3 presents the proposed problem reformulation and optimization solutions over multi-flow relaxation. Numerical simulation results are provided to validate the proposed joint cell 
association and user scheduling schemes under all kinds of carrier deployment in Section 4. Section 5 concludes the paper and discusses potential research interests.

Throughout this paper the following notations are adopted: $(x(m, n))_{M \times N}$ denotes a $M \times N$ matrix with element $x(m, n)$ on row $m$ column $n ;\lceil x\rceil_{a}^{b}$ sets boundary on variable $x$, which makes $a \leq x \leq b$. Matrices and column vectors are demonstrated by bold upper and lower case letters, respectively.

\section{Problem Statement}

In this section, a two-layer heterogeneous system overview with multiple available aggregated carrier bands is set up first, then both the network model and the signal model are introduced in detail. In order to improve the overall network capacity, a joint cell association and, finally, the carrier aggregation for traffic load balancing problem is formulated.

\subsection{System Overview}

A cellular network with multiple low power femtocell BSs underlaid macrocells is considered in this paper, where carrier aggregation is deployed to improve the overall network date rate and coverage ability on the cell edge areas (Figure 1).

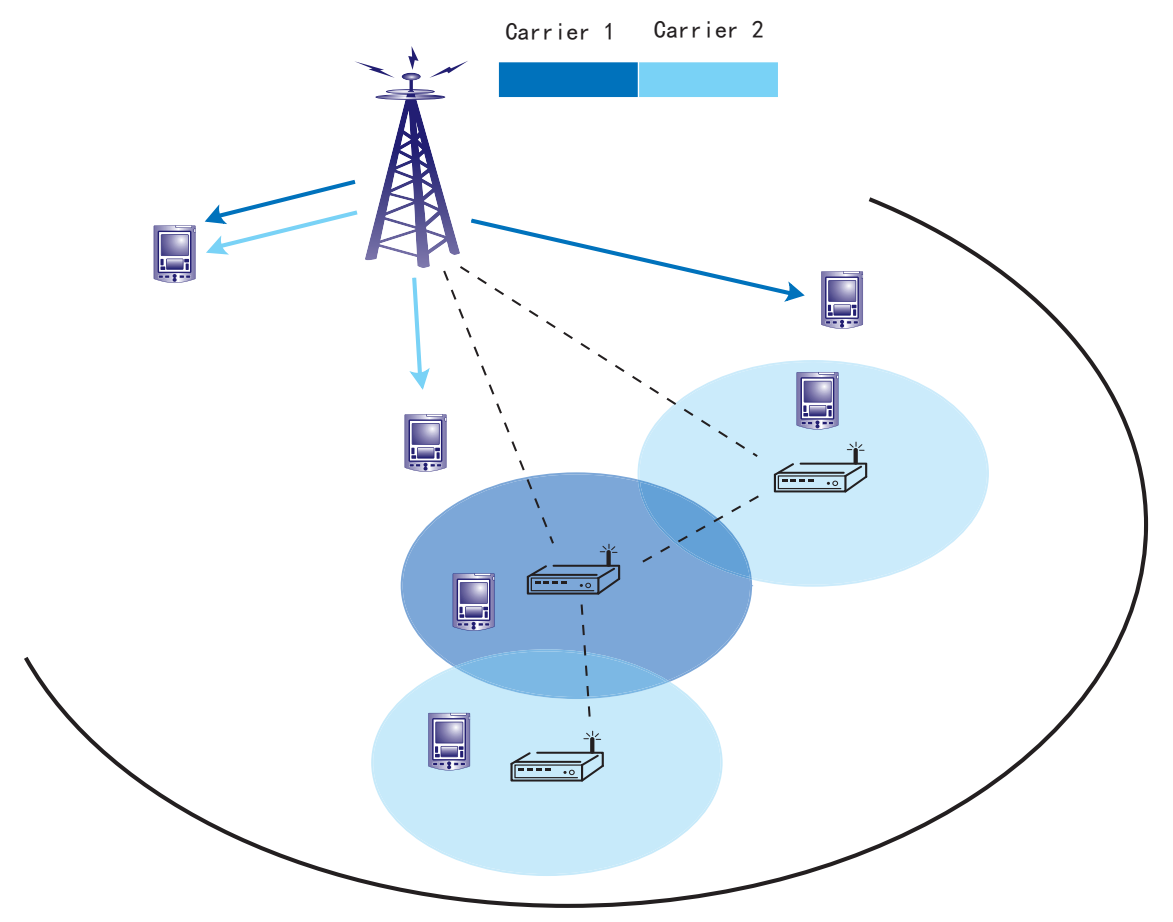

Figure 1. Carrier aggregation (CA) based Heterogenous Networks.

In this paper, a downlink scenario is considered in a multi-layer HetNet with $N$ available frequency bands denoted as a set of $\mathcal{N}=\{1, \ldots, N\}$ for carrier aggregation, and for each carrier band $n \in \mathcal{N}$, the bandwidth is denoted as $B^{n}$. Assuming a set of $\mathcal{K}=\{1, \ldots, K\}$ base stations are normally scattered in the HetNet, where massive femtocell BSs and the macrocell BS are layered by their transmit power level. A set of $\mathcal{M}=\{1, \ldots, M\}$ normally distributed users are considered active in the two-layer HetNets. 
Assume a typical downlink transmission link from BS $k \in \mathcal{K}$ to its intended user $m \in \mathcal{M}$ occupying radio resource carrier $n \in \mathcal{N}$, where $x_{k}^{n}$ is the transmit symbol. Then, the received signal at user $m$ could be denoted as

$$
y_{k, m}^{n}=\underbrace{H_{k, m}^{n} x_{k}^{n}}_{\text {Signal }}+\underbrace{\sum_{j=1, j \neq k}^{K} H_{j, m}^{n} x_{j}^{n}}_{\text {Interferences }}+\underbrace{\sigma_{n, m}}_{\text {Noise }},
$$

where $H_{k, m}^{n}$ is the complex channel state information from BS $k$ to user $m$ on carrier band $n$. The received signal at user $m$ is demonstrated as the combination of the intended signal, co-channel interferences from the neighboring cells within the HetNets and the additive white Gaussian noise (AWGN) with zero mean and variance $N_{0}$. Then, the achievable rate for user $m$ in cell $k$ scheduled on carrier $n$ should be

$$
C_{k, m}^{n}=\mathbb{E}\left\{B^{n} \log _{2}\left(1+\operatorname{SIN} R_{k, m}^{n}\right)\right\},
$$

where $\operatorname{SINR}_{k, m}^{n}$ is the signal to interference plus noise ratio accordingly. Under the assumption of signal model (1), it is denoted as

$$
\operatorname{SINR}_{k, m}^{n}=\frac{P_{k}^{n} G_{k, m}^{n}}{N_{0} B^{n}+\sum_{j=1, j \neq k}^{K} P_{j}^{n} G_{j, m}^{n}} .
$$

$P_{k}^{n}$ is the transmit power level of BS $k$ on carrier band $n$, which is denoted from $P_{k}^{n}=\mathbb{E}\left\{\left|x_{k}^{n}\right|^{2}\right\}$. $G_{k, m}^{n}=\left|H_{k, m}^{n}\right|^{2}$ is the channel power gain which captures both large and small scale fading characteristics. And $N_{0} B^{n}$ is the power level of the white Gaussian noise of bandwidth $B^{n}$.

To balance the traffic load among different tiers in the carrier aggregated HetNets, the cell association schemes are developed to determine the optimal user scheduling policy with binary indicators $\psi_{k, m}^{n}$, where

$$
\psi_{k, m}^{n}= \begin{cases}1, & \text { user } m \text { is associated with BS } k \text { on band } n \\ 0, & \text { otherwise. }\end{cases}
$$

Therefore, on each carrier band $n$, the traffic load for each BS $k$ is determined as

$$
L_{k}^{n}=\sum_{m=1}^{M} \psi_{k, m}^{n} \forall k, n
$$

where equal and orthogonal radio resource and equal power allocation is assumed among users associated with the same BS on the same carrier band in this paper.

\subsection{Problem Formulation}

As assumed above, with equal and orthogonal resource allocation, the ergodic rate of user $m$ served by BS $k$ on carrier band $n$ should be denoted as

$$
r_{k, m}^{n}=\psi_{k, m}^{n} \frac{C_{k, m}^{n}}{L_{k}^{n}}
$$

which is depending on not only the scheduling indicator but also the traffic load of the scheduled service supplier for each user.

In the traditional cellular network, to bring the best performance, users are always connected to the BSs who would provide with the best Signal to Interference plus Noise Ratio (SINR) performances. In the two-layer HetNets, due to the different power levels between the macro Bs and the Femto 
BSs, it is always the Macro BS that could serve the users under such cell association policies. Thereby, the traffic load would be extremely uneven among different layers causing the over loaded macrocell and lightly loaded femtocells, which has prevented the heterogeneous network from achieving better performances.

To balance the traffic load and maximize the network utility, cell association schemes are designed that aim to determine the optimal user scheduling policy

$$
\begin{array}{ll}
\max _{\mathbf{\Psi}, \mathbf{L}} & \sum_{n=1}^{N} \sum_{k=1}^{K} \sum_{m=1}^{M} r_{k, m}^{n} \\
\text { s.t. } & \sum_{k=1}^{K} \sum_{n=1}^{N} \psi_{k, m}^{n}=1, \forall m, \\
& L_{k}^{n}=\sum_{m=1}^{M} \psi_{k, m}^{n} \leq M, \forall k, n, \\
& \psi_{k, m}^{n} \in\{0,1\}, \forall k, n, m,
\end{array}
$$

where $\Psi=\left(\psi_{k, m}^{n}\right)_{k \in \mathcal{K}, m \in \mathcal{M}}^{n \in \mathcal{N}}, \boldsymbol{L}=\left(L_{k}^{n}\right)_{k \in \mathcal{K}}^{n \in \mathcal{N}}$ and the network utility function is defined as the sum rate of the whole network.

Problems such as the above are nonlinear and non-convex combinatorial optimization problems for which it is unpractical to search for a global solution [36]. In this paper, two load balanced cell association schemes are involved on the basis of carrier aggregation techniques for the two-tier HetNets. In an earlier work of ours, a distributed stochastic control based [37] user scheduling scheme is proposed for single flow carrier aggregation where users can only associate with no more than one $\mathrm{BS}$ on one carrier. In this paper, the problem is reformulated to the circumstances where multiple flows are allowed for carrier aggregation to achieve a joint solution across the HetNets.

\section{Joint Cell Association Scheme for Multi-Flow Carrier Aggregation}

In this section, joint cell association is proposed for multi-flow carrier aggregation across different BS in different layers, which may provide with an upper bound on the network throughput maximization problem.

\subsection{Multi-Flow Problem Reformulation}

In this part, for each user it is assumed that they could be associated with multiple BSs on different carriers at one time, which indicates that the constraint on $\psi_{k, m}^{n} \in\{0,1\}$ has been relaxed to $\psi_{k, m}^{n} \in[0,1]$. As long as $\psi_{k, m}^{n}>0$, user $m$ will be fractionally scheduled on carrier band $n$ by BS $k$, with regard to which, a single flow problem is relaxed to a multi flow problem formulation.

In order to achieve better performance on traffic load balancing across different layers of the HetNets, logarithmic utility is considered instead of the linear utility in (8). With such considerations, fairness among users are better covered by the concaveness of the logarithm, which also helps to balance the traffic between different tiers.

Therefore, under equal resource allocation assumption among all of the users, the joint cell association problem is reformulated as

$$
\begin{array}{ll}
\max _{\Psi, L} & \sum_{n=1}^{N} \sum_{k=1}^{K} \sum_{m=1}^{M} \psi_{k, m}^{n} \log \left(\frac{C_{k, m}^{n}}{L_{k}^{n}}\right) \\
\text { s.t. } & \sum_{k=1}^{K} \sum_{n=1}^{N} \psi_{k, m}^{n}=1, \forall m, \\
& 0 \leq L_{k}^{n}=\sum_{m=1}^{M} \psi_{k, m}^{n} \leq M, \forall k, n, \\
& 0 \leq \psi_{k, m}^{n} \leq 1, \forall k, n, m,
\end{array}
$$

where $\Psi=\left(\psi_{k, m}^{n}\right)_{k \in \mathcal{K}, m \in \mathcal{M}}^{n \in \mathcal{N}}$ and $\boldsymbol{L}=\left(L_{k}^{n}\right)_{k \in \mathcal{K}}^{n \in \mathcal{N}}$. 


\subsection{Iterative Dual Analysis Based Joint Cell Association}

The relaxed problem reformulation (9) has changed the proposed problem (8) from combinatorial optimization into convex optimization. The original problem could be considered as a special case where the users are all associated with only one BS on one carrier band.

To solve this problem, the Lagrangian Dual Decomposition approach [36] is involved after (9) is rewritten as

$$
\begin{aligned}
& \max _{\mathbf{\Psi}, \mathbf{L}} \sum_{n=1}^{N} \sum_{k=1}^{K} \sum_{m=1}^{M} \psi_{k, m}^{n}\left(\log \left(C_{k, m}^{n}\right)-\log \left(L_{k}^{n}\right)\right) \\
& =\max _{\Psi, L} \sum_{n=1}^{N} \sum_{k=1}^{K} \sum_{m=1}^{M} \psi_{k, m}^{n} \log \left(C_{k, m}^{n}\right)-\sum_{n=1}^{N} \sum_{k=1}^{K} L_{k}^{n} \log \left(L_{k}^{n}\right) \\
& \text { s.t. } \quad \sum_{k=1}^{K} \sum_{n=1}^{N} \psi_{k, m}^{n}=1, \forall m \text {, } \\
& L_{k}^{n}=\sum_{m=1}^{M} \psi_{k, m^{\prime}}^{n} \quad \forall k, n, \\
& 0 \leq \psi_{k, m}^{n} \leq 1, \forall k, n, m, \\
& 0 \leq L_{k}^{n} \leq M, \forall k, n ，
\end{aligned}
$$

where $\boldsymbol{\Psi}=\left(\psi_{k, m}^{n}\right)_{k \in \mathcal{K}, m \in \mathcal{M}}^{n \in \mathcal{N}}$ and $\boldsymbol{L}=\left(L_{k}^{n}\right)_{k \in \mathcal{K}}^{n \in \mathcal{K}}$.

The Lagrangian function is constructed first as

$$
\begin{aligned}
\Lambda(\Psi, L, \lambda)= & \sum_{n=1}^{N} \sum_{k=1}^{K} \sum_{m=1}^{M} \psi_{k, m}^{n} \log \left(C_{k, m}^{n}\right) \\
& -\sum_{k=1}^{K} \sum_{n=1}^{N} L_{k}^{n} \log \left(L_{k}^{n}\right) \\
& +\sum_{n=1}^{N} \sum_{k=1}^{K} \lambda_{k}^{n}\left(L_{k}^{n}-\sum_{m=1}^{M} \psi_{k, m}^{n}\right)
\end{aligned}
$$

where $\lambda=\left(\lambda_{k}^{n}\right)_{K \times N}$ is constructed as the Lagrangian multipliers.

Then, the optimization problem (10) is dualized by defining this dual problem

$$
\begin{array}{ll}
g(\boldsymbol{\lambda}) & =\max \Lambda(\boldsymbol{\Psi}, \boldsymbol{L}, \boldsymbol{\lambda}) \\
\text { s.t. } & \sum_{k=1}^{K} \sum_{n=1}^{N} \psi_{k, m}^{n}=1, \forall m, \\
& 0 \leq L_{k}^{n}=\sum_{m=1}^{M} \psi_{k, m}^{n} \leq M, \forall k, n, \\
& 0 \leq \psi_{k, m}^{n} \leq 1, \forall k, n, m,
\end{array}
$$

As it could be inferred from (11) and (12) that the dual problem is composed of two independent subproblems illustrated as

$$
\begin{aligned}
& \max _{\Psi} \sum_{n=1}^{N} \sum_{k=1}^{K} \sum_{m=1}^{M} \psi_{k, m}^{n} \log \left(C_{k, m}^{n}\right)-\sum_{n=1}^{N} \sum_{k=1}^{K} \lambda_{k}^{n} \sum_{m=1}^{M} \psi_{k, m}^{n} \\
& =\max _{\Psi} \sum_{n=1}^{N} \sum_{k=1}^{K} \sum_{m=1}^{M} \psi_{k, m}^{n}\left(\log \left(C_{k, m}^{n}\right)-\lambda_{k}^{n}\right) \\
& \text { s.t. } \quad \sum_{k=1}^{K} \sum_{n=1}^{N} \psi_{k, m}^{n}=1, \forall m \text {, } \\
& 0 \leq \psi_{k, m}^{n} \leq 1, \quad \forall k, n, m \text {, }
\end{aligned}
$$


where $\Psi=\left(\psi_{k, m}^{n}\right)_{k \in \mathcal{K}, m \in \mathcal{M}}^{n \in \mathcal{N}}$, and

$$
\begin{aligned}
\max _{L} & \sum_{n=1}^{N} \sum_{k=1}^{K} \lambda_{k}^{n} L_{k}^{n}-\sum_{n=1}^{N} \sum_{k=1}^{K} L_{k}^{n} \log \left(L_{k}^{n}\right) \\
=\max _{L} & \sum_{n=1}^{N} \sum_{k=1}^{K} L_{k}^{n}\left(\lambda_{k}^{n}-\log \left(L_{k}^{n}\right)\right) \\
\text { s.t. } & L_{k}^{n}=\sum_{m=1}^{M} \psi_{k, m}^{n}, \forall k, n, \\
& 0 \leq L_{k}^{n} \leq M, \forall k, n,
\end{aligned}
$$

where $\boldsymbol{L}=\left(L_{k}^{n}\right)_{k \in \mathcal{K}}^{n \in \mathcal{K}}$.

This dual formulation has already proved by Slater's condition [36] to have a zero dual gap with the primal problem when the optimal resolution of (13) and (14) converges into the same, which means that solving the dual problem (12) offers the original problem (9) with an optimal solution.

For each $\lambda_{k}^{n}$, the optimal determination of $\psi_{k, m}^{n}$ in (13) is to determine which user should be associated with which BS on which carrier which is

$$
f^{*}(m)=\arg \max _{(k, n)}\left(\log \left(C_{k, m}^{n}\right)-\lambda_{k}^{n}\right), \quad \forall m .
$$

Apply the well known Karush-Kuhn-Tucker (KKT) method on problem (14) and get

$$
L_{k}^{n}=\left\lceil e^{\lambda_{k}^{n}-1}\right\rceil_{0}^{M} .
$$

To summarize, searching for the maximum sum rate is proceeded by two layers of iterations. The first layer is implemented between the optimal results of (13) and (14). The second layer is the rate adaptation along with the updation of the Lagrangian multipliers. The second layer iteration takes place between the Lagrangian multipliers $\lambda$ and the converged sum rate achieved from the first layer iteration. The subgradient method is used for the updation of $\lambda$ as for any $k$ or $n$ there is

$$
\lambda_{k}^{n}(\text { Iter }+1)=\left\lceil\lambda_{k}^{n}(\text { Iter })-s(\text { Iter })\left(L_{k}^{n}-\sum_{m=1}^{M} \psi_{k, m}^{n}\right)\right]_{0}^{+},
$$

where Iter is the iteration number and $s$ is the iteration step chosen by the subgradient method [36].

\subsection{Summary}

The pseudo code of the proposed joint cell association scheme with multiple flow CA is summarized as in Algorithm 1. 


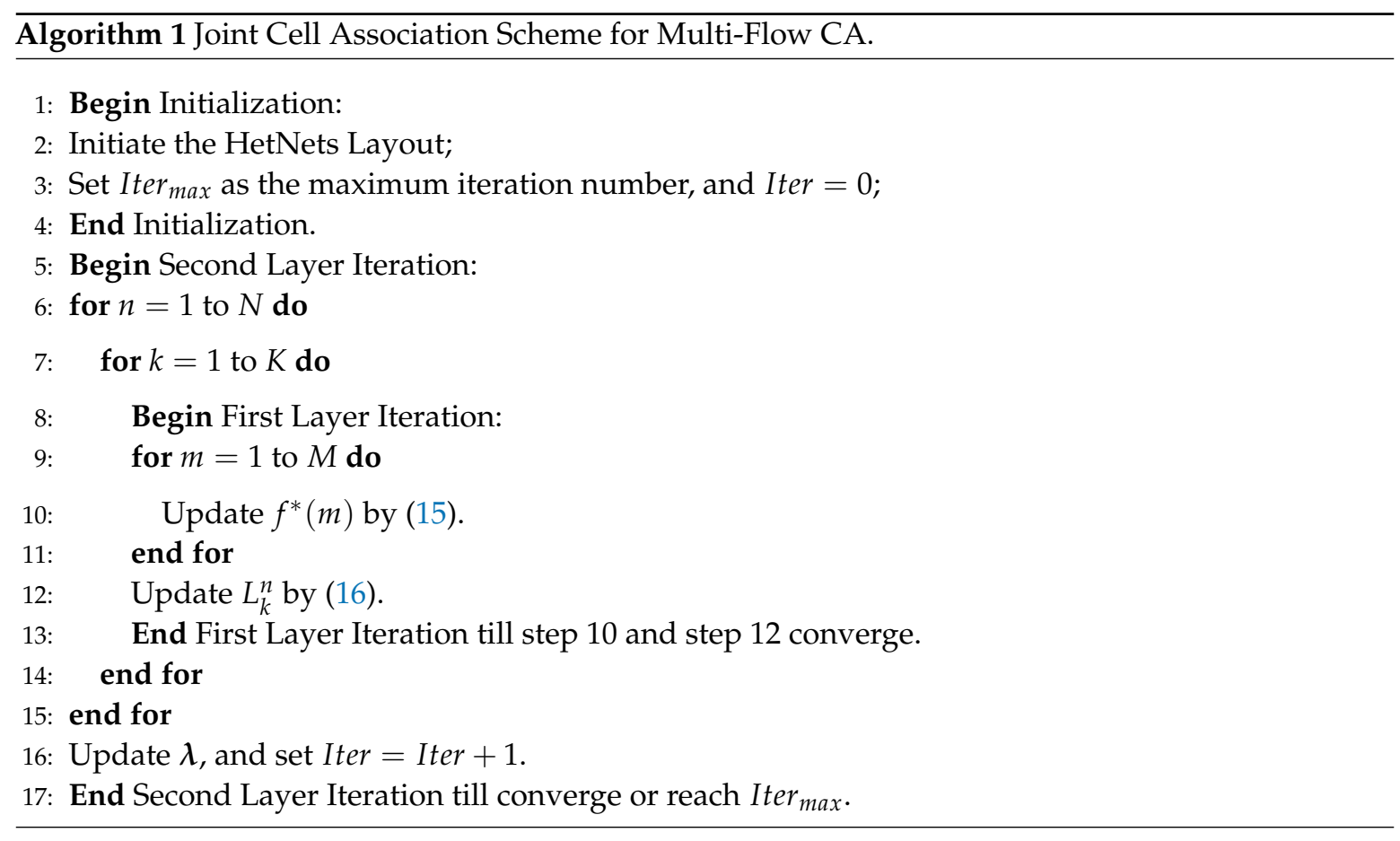

\section{Numerical Analysis}

In this section, simulation results are demonstrated to evaluate the performances of the proposed multi-flow based joint cell association schemes on the traffic load balancing and the network utility problems.

\subsection{Simulation Configuration}

In a typical two layer Heterogeneous Network, the carrier bands for aggregation is set as $N=2$, with one high frequency carrier band $C^{1}$ and one low frequency carrier band $C^{2}$. The carrier bands deployments are denoted by a binary matrix between different network layers and different carrier frequencies as in $\left[\alpha_{11} \alpha_{12} ; \alpha_{21} \alpha_{22}\right]$, where

$$
\alpha_{i_{1} i_{2}}= \begin{cases}1, & \text { carrier } i_{2} \text { is deployed on HetNet layer } i_{1} \\ 0, & \text { otherwise. }\end{cases}
$$

For example, a deployment of [1 0;1 1] means the first tier only has access to carrier $C^{1}$, and the second tier is co-deployed with both carrier bands $C^{1}$ and $C^{2} c$. In this simulation set-ups, the first layer is referring to the macrocell layer, and the second is femtocell layer.

As stated in Section 2, both small scale and large scale fading effects are considered in the channel power gain $G_{k, m}^{n}$ from (3). The small scale Rayleigh fading effects in $G_{k, m}^{n}$ is stated by a random variable with zero mean and unit variance, while the large scale fading is assumed by only denoting the path loss effect. A simplified path loss model [38] is built as a function of distance between a user and it serves BS as follows

$$
P L\left(d_{k, m}\right)=C_{n}\left(\frac{d_{0}}{d_{k, m}}\right)^{\gamma} .
$$

In this model, the variable $d_{k, m}$ denotes the distance between user $m$ and BS $k . C_{n}$ is a frequency-dependent constant denoted as $C_{n}=\left(\lambda_{n} / 4 \pi d_{0}\right)^{2}$, where $d_{0}$ is the reference distance for the antenna far field, and $\lambda_{n}$ is the wavelength of radio carrier $n \in \mathcal{N}$. And $\gamma$ is the path-loss exponent depending on propagation environment. Note that considering the scattering environment, this path loss model is valid only when $d_{k, m}>d_{0}$ [38]. 
The other simulation parameters are summarized in Table 1 unless stated otherwise. All of the simulation results are obtained through at least 1000 Monte Carlo channel realizations. For comparison, a stochastic single-flow solution [37] and the traditional max-SINR cell association strategy are considered as benchmarks in the simulation results.

Table 1. Simulation Parameter Statements.

\begin{tabular}{cc}
\hline Macrocell Transmit Power & $46 \mathrm{dBm}$ \\
\hline Femtocell Transmit Power & $20 \mathrm{dBm}$ \\
\hline Macrocell coverage Radius & $500 \mathrm{~m}$ \\
\hline Femtocell coverage Radius & $50 \mathrm{~m}$ \\
\hline AWGN $N_{0}$ & $-174 \mathrm{dBm} / \mathrm{Hz}$ \\
\hline Total Users Number & $\mathrm{M}=200$ \\
\hline Carrier Frequency of $C^{1}$ & $2.5 \mathrm{GHz}$ \\
\hline Carrier Frequency of $C^{2}$ & $800 \mathrm{MHz}$ \\
\hline Bandwidth $B^{1}$ of $C^{1}$ & $10 \mathrm{MHz}$ \\
\hline Bandwidth $B^{2}$ of $C^{2}$ & $10 \mathrm{MHz}$ \\
\hline Wavelength $\lambda_{1}$ of $C^{1}$ & $0.125 \mathrm{~m}$ \\
\hline Wavelength $\lambda_{2}$ of $C^{2}$ & $0.375 \mathrm{~m}$ \\
\hline Path Loss Exponent $\gamma^{1}$ of Macrocell & 4 \\
\hline Path Loss Exponent $\gamma^{2}$ of Femtocell & 3 \\
\hline Reference distance $d_{0}$ in Macrocell & $10 \mathrm{~m}$ \\
\hline Reference distance $d_{0}$ in Femtocell & $1 \mathrm{~m}$ \\
\hline
\end{tabular}

\subsection{Numerical Results}

Numerical results are demonstrated in this section under pre-discussed network layout and system parameters under different carrier deployments.

Figure 2 shows the sum-rate performance of the proposed joint cell association schemes for multi-flow CA when the total number of users within the HetNets are changing under different carrier bands deployments. The proposed multi-flow solution (MF) is compared with the Max-SINR (SINR) association scheme under different network density, where $K=20$ and $K=40$ state the total BS number in the network layout. It can be seen that, under different carrier deployment conditions, the proposed joint scheme is proved to have much better performance in terms of network capacity. Compared with the previous work [37], under the same condition of carrier deployment, the proposed joint scheme is also better than the stochastic single flow scheme due to the user diversity gain. The relaxation of the user association factor $\psi_{k, m}^{n}$ makes the users capable of associating with multiple BSs on multiple carriers in the same time and in the mean time, the global solution is achieved.

When carrier deployment changes among the two-layer HetNets, Figures 3-5 show the performance on network capacity against the transmit power lever of both Macro BS and Femto BSs. In general, the proposed joint solution gives the best performance as expected. According to different carrier deployment conditions, the performance differences are summarized below in detail.

Figure 3 indicates the situation in which the first layer of the HetNets is employed with both high and low frequency bands while the second layer is left with a single carrier band. In Figure $3 a$, as the growth of the transmit power of Macro BS, the proposed joint solution achieves better performance due to its designed multiple data flow cell association scheme. For the other two schemes, the users scheduled in the second layer suffered severely from the growing interference level, which blocks the whole network performances from improving. From Figure 3b, it could be seen that the proposed joint scheme is more sensitive to the transmit power level of the Femto BS compared to the other two 
scheme. As the transmit power grows, the users are offloaded from the Macro BS, which causes the network utility improving. However, as the power level continues to grow, the cross layer interference has become a threat to the HetNets, which causes the performance drop. This precisely proves the effectiveness and flexibility and effectiveness of the proposed joint scheme.

Under different circumstances in Figure 4, the proposed solution along with the two benchmark schemes have shown their own characteristics when cross layer interferences exists([1 1;1 1], [0 1;1 1], [1 0;1 1]). In Figure 4a, the proposed joint multi-flow solution achieves the best performance under full carrier deployment across different network layers ([1 $1 ; 11])$. The other two schemes both suffer a performance loss as the interference level grows either inter layer or inner layer. As in Figure $4 \mathrm{~b}$, under full carrier deployment, the proposed solution only achieves better performance when the transmit power of the Femto BSs reaches a certain level. When the transmit powers are lower, the stochastic single flow solution provides the HetNets with better utility because there are more available BSs and carrier bands in the Femtocells for each user to choose a better cell association opportunity.

In particular, when cross layer interferences do not exist in Figure 5 ([1 0;0 1], [0 1;1 0]), the transmit power of both Macro BS and Femto BS does not have the same impacts on the network utility as the other carrier deployment circumstances for all three schemes.

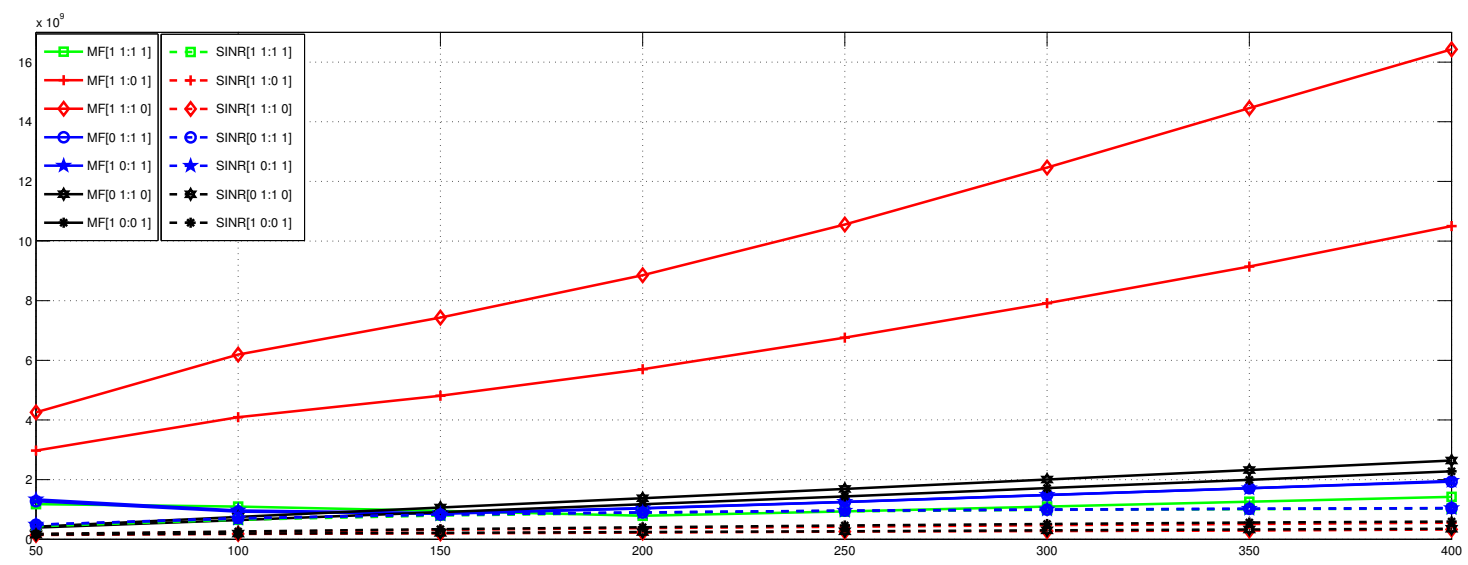

(a)

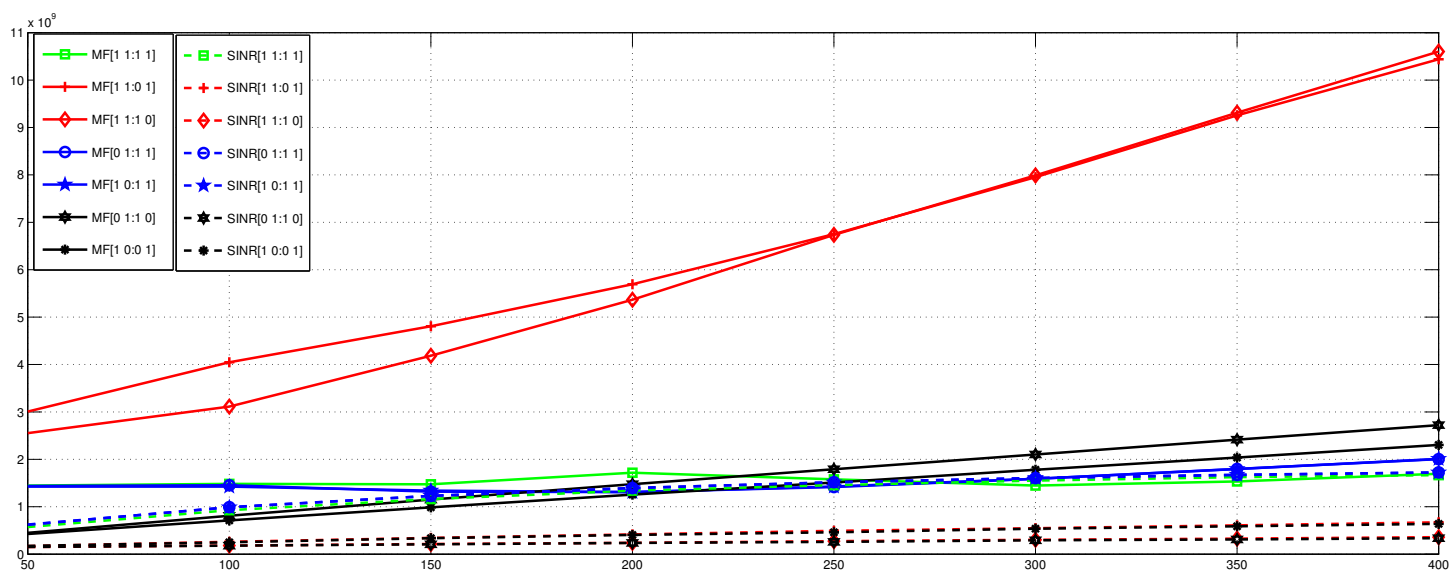

$(\mathbf{b})$

Figure 2. Network Sum Rate against User Number for Multi-Flow CA. (a) $K=20$; (b) $K=40$. 


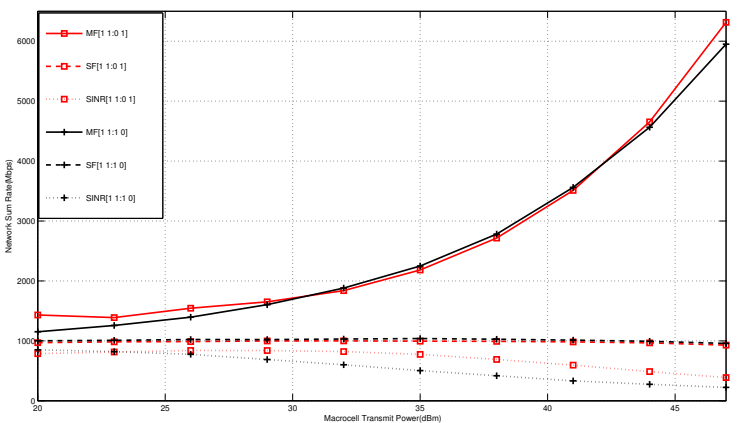

(a)

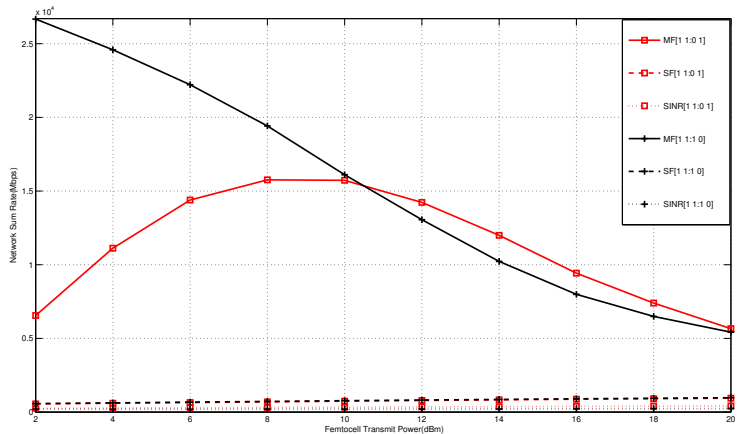

(b)

Figure 3. Network Sum Rate against Transmit Power with CA deployment. $(K=40, M=200)$. (a) Macrocell Transmit Power; (b) Femtocell Transmit Power.

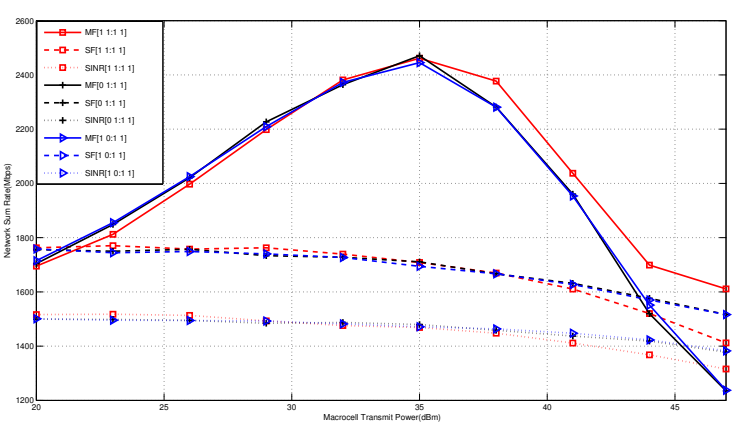

(a)

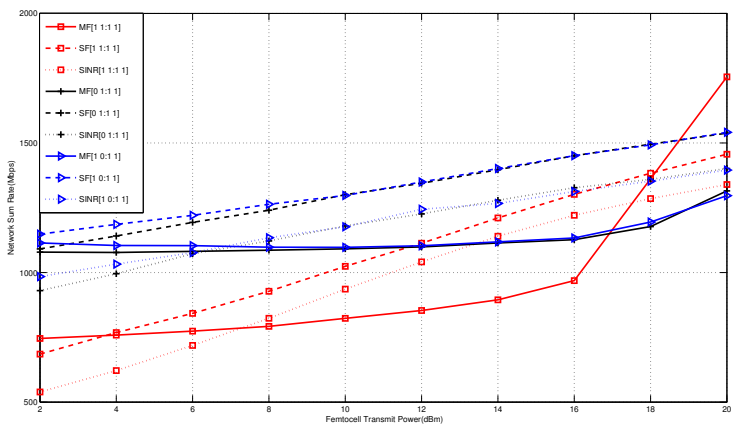

(b)

Figure 4. Network Sum Rate against Transmit Power with CA deployment. $(K=40, M=200)$. (a) Macrocell Transmit Power; (b) Femtocell Transmit Power.

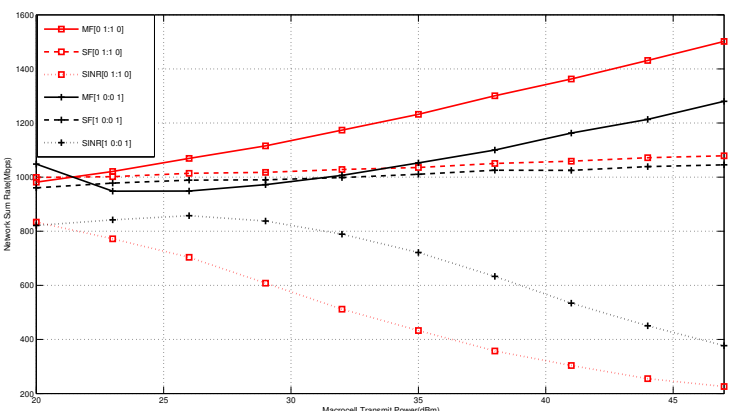

(a)

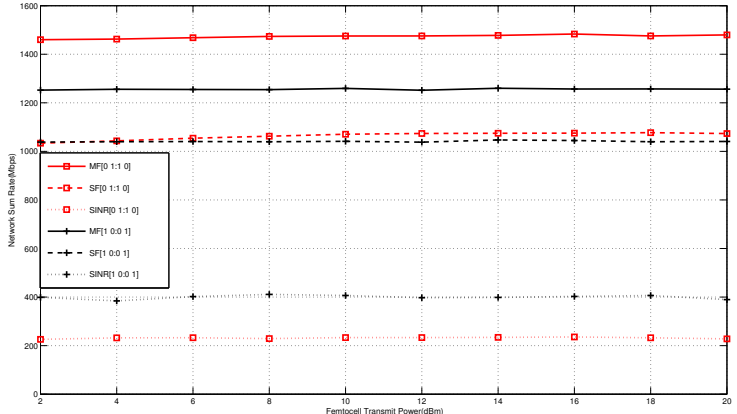

(b)

Figure 5. Network Sum Rate against Transmit Power with CA deployment. $(K=40, M=200)$. (a) Macrocell Transmit Power; (b) Femtocell Transmit Power.

\section{Conclusions}

This paper approaches the network capacity maximization problem through joint cell association and user scheduling in the HetNet. With multiple carrier aggregated across different layers for the downlink transmission, the original joint maximization problem is reformulated, in which the users could associate with BSs on more than one carrier band. The flexibility of the proposed joint maximization problem was achieved by convex optimization solutions with reasonable complexity. Numerical results were able to show the performance advantages of the proposed multi-flow solution under different carrier aggregation deployment. 
For future work, the joint cell association and user scheduling problem in the HetNets could be considered with physical layer multiple, or even massive, antennas involved in the transmission design, where the users may access the network non-orthogonally by multiplexing in the power domain.

Acknowledgments: The work in this paper was supported by National Natural Science Foundation of China (Grants No. 61601269, No. 61402268 and No. 61701269), and Shandong Natural Science Foundation (Grants No. ZR2015PF006).

Author Contributions: Jia Zhang proposed the idea, carried out the simulations and wrote the paper; Lili Meng and Hui Ji analyzed the data; all authors have read and approved the final manuscript.

Conflicts of Interest: The authors declare no conflict of interest.

\section{References}

1. Andrews, J.G.; Buzzi, S.; Choi, W.; Hanly, S.V.; Lozano, A.; Soong, A.C.K.; Zhang, J.C. What Will 5G Be? IEEE J. Sel. Areas Commun. 2014, 32, 1065-1082, doi:10.1109/JSAC.2014.2328098.

2. Soret, B.; Wang, H.; Pedersen, K.I.; Rosa, C. Multicell Cooperation for LTE-advanced Heterogeneous Network Scenarios. IEEE Wirel. Commun. 2013, 20, 27-34.

3. Damnjanovic, A.; Montojo, J.; Wei, Y.; Ji, T.; Luo, T.; Vajapeyam, M.; Yoo, T.; Song, O.; Malladi, D. A Survey on 3GPP Heterogeneous Networks. IEEE Wirel. Commun. 2011, 18, 10-21.

4. Dhillon, H.S.; Ganti, R.K.; Baccelli, F.; Andrews, J.G. Modeling and Analysis of K-Tier Downlink Heterogeneous Cellular Networks. IEEE J. Sel. Areas Commun. 2012, 30, 550-560, doi:10.1109/JSAC.2012.120405.

5. Lopez-Perez, D.; Chu, X.; Guvenc, İ. On the Expanded Region of Picocells in Heterogeneous Networks. IEEE J. Sel. Top. Signal Process. 2012, 6, 281-294.

6. Papadogiannis, A.; Alexandropoulos, G.C. The value of dynamic clustering of base stations for future wireless networks. In Proceedings of the International Conference on Fuzzy Systems, Barcelona, Spain, 18-23 July 2010; pp. 1-6.

7. Lee, J.; Kim, Y.; Lee, H.; Ng, B.L.; Mazzarese, D.; Liu, J.; Xiao, W.; Zhou, Y. Coordinated multipoint transmission and reception in LTE-advanced systems. IEEE Commun. Mag. 2012, 50, 44-50.

8. Alexandropoulos, G.C.; Ferrand, P.; Gorce, J.M.; Papadias, C.B. Advanced coordinated beamforming for the downlink of future LTE cellular networks. IEEE Commun. Mag. 2016, 54, 54-60.

9. Alexandropoulos, G.C.; Ferrand, P.; Papadias, C.B. On the Robustness of Coordinated Beamforming to Uncoordinated Interference and CSI Uncertainty. In Proceedings of the 2017 IEEE Wireless Communications and Networking Conference (WCNC), San Francisco, CA, USA, 19-22 March 2017; pp. 1-6.

10. Jungnickel, V.; Manolakis, K.; Zirwas, W.; Panzner, B.; Braun, V.; Lossow, M.; Sternad, M.; Apelfrojd, R.; Svensson, T. The role of small cells, coordinated multipoint, and massive MIMO in 5G. IEEE Commun. Mag. 2014, 52, 44-51.

11. Boccardi, F.; Heath, R.W.; Lozano, A.; Marzetta, T.L.; Popovski, P. Five disruptive technology directions for 5G. IEEE Commun. Mag. 2014, 52, 74-80.

12. Saquib, N.; Hossain, E.; Le, L.B.; Kim, D.I. Interference management in OFDMA femtocell networks: Issues and approaches. IEEE Wirel. Commun. 2012, 19, 86-95.

13. Zahir, T.; Arshad, K.; Nakata, A.; Moessner, K. Interference Management in Femtocells. IEEE Commun. Surv. Tutor. 2013, 15, 293-311, doi:10.1109/surv.2012.020212.00101.

14. Deb, S.; Monogioudis, P.; Miernik, J.; Seymour, J.P. Algorithms for Enhanced Inter-Cell Interference Coordination (eICIC) in LTE HetNets. IEEE/ACM Trans. Netw. 2014, 22, 137-150.

15. Fooladivanda, D.; Rosenberg, C. Joint Resource Allocation and User Association for Heterogeneous Wireless Cellular Networks. IEEE Trans. Wirel. Commun. 2013, 12, 248-257.

16. Ghimire, J.; Rosenberg, C. Resource Allocation, Transmission Coordination and User Association in Heterogeneous Networks: A Flow-Based Unified Approach. IEEE Trans. Wirel. Commun. 2013, 12, 1340-1351.

17. Chen, Y.; Li, J.; Chen, W.; Lin, Z.; Vucetic, B. Joint User Association and Resource Allocation in the Downlink of Heterogeneous Networks. IEEE Trans. Veh. Technol. 2016, 65, 5701-5706.

18. Sanjabi, M.; Razaviyayn, M.; Luo, Z.Q. Optimal Joint Base Station Assignment and Beamforming for Heterogeneous Networks. IEEE Trans. Signal Process. 2014, 62, 1950-1961. 
19. Sun, R.; Hong, M.; Luo, Z.Q. Joint Downlink Base Station Association and Power Control for Max-Min Fairness: Computation and Complexity. IEEE J. Sel. Areas Commun. 2015, 33, 1040-1054.

20. Shen, K.; Liu, Y.F.; Ding, D.Y.; Yu, W. Flexible Multiple Base Station Association and Activation for Downlink Heterogeneous Networks. IEEE Signal Process. Lett. 2017, 24, 1498-1502.

21. Ye, Q.; Rong, B.; Chen, Y.; Al-Shalash, M.; Caramanis, C.; Andrews, J.G. User Association for Load Balancing in Heterogeneous Cellular Networks. IEEE Trans. Wirel. Commun. 2013, 12, 2706-2716, doi:10.1109/twc.2013.040413.120676.

22. Gupta, A.K.; Dhillon, H.S.; Vishwanath, S.; Andrews, J.G. Downlink Multi-Antenna Heterogeneous Cellular Network With Load Balancing. IEEE Trans. Commun. 2014, 62, 4052-4067.

23. Shen, K.; Yu, W. Load and Interference Aware Joint Cell Association and User Scheduling in Uplink Cellular Networks. In Proceedings of the 2016 IEEE 17th International Workshop on Signal Processing Advances in Wireless Communications (SPAWC), Edinburgh, UK, 3-6 July 2016; pp. 1-5.

24. Lin, Y.; Bao, W.; Yu, W.; Liang, B. Optimizing User Association and Spectrum Allocation in HetNets: A Utility Perspective. IEEE J. Sel. Areas Commun. 2015, 33, 1025-1039.

25. Zhang, Z.; Song, L.; Han, Z.; Saad, W. Coalitional Games with Overlapping Coalitions for Interference Management in Small Cell Networks. IEEE Trans. Wirel. Commun. 2014, 13, 2659-2669.

26. Saad, W.; Han, Z.; Zheng, R.; Debbah, M.; Poor, H.V. A College Admissions Game for Uplink User Association in Wireless Small Cell Networks. In Proceedings of the IEEE INFOCOM 2014-IEEE Conference on Computer Communications, Toronto, ON, Canada, 27 April-2 May 2014; pp. 1096-1104.

27. Ngo, D.T.; Khakurel, S.; Le-Ngoc, T. Joint Subchannel Assignment and Power Allocation for OFDMA Femtocell Networks. IEEE Trans. Wirel. Commun. 2014, 13, 342-355, doi:10.1109/TWC.2013.111313.130645.

28. Lee, H.; Vahid, S.; Moessner, K. A Survey of Radio Resource Management for Spectrum Aggregation in LTE-Advanced. IEEE Commun. Surv. Tutor. 2014, 16, 745-760.

29. Fazliu, Z.L.; Chiasserini, C.F.; Dell'Aera, G.M.; Hamiti, E. Distributed Downlink Power Control for Dense Networks With Carrier Aggregation. IEEE Trans. Wirel. Commun. 2017, 16, 7052-7065.

30. Lin, X.; Andrews, J.G.; Ghosh, A. Modeling, Analysis and Design for Carrier Aggregation in Heterogeneous Cellular Networks. IEEE Trans. Commun. 2013, 61, 4002-4015.

31. Bhamri, A.; Hooli, K.; Lunttila, T. Massive Carrier Aggregation in LTE-advanced Pro: Impact on Uplink Control Information and Corresponding Enhancements. IEEE Commun. Mag. 2016, 54, 92-97.

32. Stefanatos, S.; Foukalas, F.; Tsiftsis, T.A. Low Complexity Resource Allocation for Massive Carrier Aggregation. IEEE Trans. Veh. Technol. 2017, 66, 9614-9619.

33. Shajaiah, H.; Abdelhadi, A.; Clancy, C. Robust Resource Allocation with Joint Carrier Aggregation in Multi-Carrier Cellular Networks. IEEE Trans. Cogn. Commun. Netw. 2017, doi:10.1109/TCCN.2017.2773082.

34. Liao, H.S.; Chen, P.Y.; Chen, W.T. An Efficient Downlink Radio Resource Allocation with Carrier Aggregation in LTE-Advanced Networks. IEEE Trans. Mobile Comput. 2014, 13, 2229-2239.

35. Tsinos, C.G.; Foukalas, F.; Tsiftsis, T.A. Resource Allocation for Licensed/Unlicensed Carrier Aggregation MIMO Systems. IEEE Trans. Commun. 2017, 65, 3765-3779.

36. Boyd S, Vandenberghe, L. Convex Optimization; Cambridge University Press: Cambridge, UK, 2014.

37. Zhang, J.; Yuan, D.; Zhang, H. On Stochastic Cell Association Scheme Over Carrier Aggregated Heterogenous Networks. J. Comput. 2013, 8, 2895-2901.

38. Goldsmith, A. WIreless Communications; Cambridge University Press: Cambridge, UK, 2005.

(C) 2018 by the authors. Licensee MDPI, Basel, Switzerland. This article is an open access article distributed under the terms and conditions of the Creative Commons Attribution (CC BY) license (http://creativecommons.org/licenses/by/4.0/). 